\title{
Hybrid morphology radio sources from the FIRST survey ${ }^{\star}$
}

\author{
M. P. Gawroński, A. Marecki, M. Kunert-Bajraszewska, and A. J. Kus
}

Toruń Centre for Astronomy, N. Copernicus University, 87-100 Toruń, Poland

e-mail: amr@astro.uni.torun.pl

Received 5 August 2005 / Accepted 13 September 2005

\section{ABSTRACT}

The so-called HYbrid MOrphology Radio Sources (HYMORS) are a class of objects that appear to have a mixed Fanaroff-Riley (FR) morphology in a single object; i.e. a HYMORS has an FR I-type lobe on one side of its nucleus and an FR II-type lobe on the other side. Because of this unique feature and given that the origin of the FR morphological dichotomy is still unclear, HYMORS may possibly play a crucial role in our understanding of the FR-dichotomy. As the number of known HYMORS is quite small, we aimed to increase that number by inspecting a few areas of the sky covered by the VLA FIRST survey and by selecting 21 HYMORS candidates based on the morphology shown in the FIRST images. They were observed with the VLA in B-conf. at $4.9 \mathrm{GHz}$. Three objects from the initial sample turned out to be actual HYMORS and two others very likely to fulfill the criteria. These five were subsequently re-observed with the VLA in A-conf. at $1.4 \mathrm{GHz}$. Our results provide strong support to the findings of Gopal-Krishna \& Wiita (2000, A\&A, 363, 507), namely that there are two different kinds of jets in HYMORS; consequently, the existence of FR-dichotomy as a whole is difficult to reconcile with the class of explanations that posit fundamental differences in the central engine.

Key words. radio continuum: galaxies - galaxies: active - galaxies: jets - galaxies: nuclei

\section{Introduction}

Fanaroff \& Riley (1974) have shown that there are two distinct morphological classes of double radio galaxies and that there is a relatively sharp transition across a critical radio luminosity $L_{\mathrm{R}}^{\star}$, corresponding to $L_{178 \mathrm{MHz}} \simeq 5 \times 10^{24} h^{-2} \mathrm{~W} \mathrm{~Hz}^{-1} \mathrm{sr}^{-1}$. Most sources below this luminosity threshold belong to the FR I class of radio galaxies. FR I structure consists of diffuse radio lobes that have their brightest regions within the inner parts of the radio source. The cores are usually prominent in FR I sources and they often show bent jets; in these cases FR I objects can be labelled the Wide Angle Tail, Narrow Angle Tail, and head-tail sources. More powerful FR II-type double sources have their hotspots at the outer edges of the two radio lobes, and typically only one jet is clearly detectable. The cores of these sources are weak or hardly observable. There is strong evidence that FR I and FR II sources undergo different cosmological evolution (Wall 1980); i.e. the density and/or luminosity evolution rate of FR II-type is high, while that of FR I appears to be low. It is to be noted that $L_{\mathrm{R}}^{\star}$ is found to be near a transition in the properties of nuclear optical emission lines (Hine \& Longair 1979).

The origin of FR I/FR II-dichotomy is much debated in the astrophysics of extragalactic radio sources. There are three main ways of interpreting it:

- morphological differences are related to the transition of an initially supersonic but relatively weak jet to a

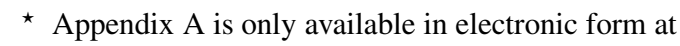
http://www. edpsciences.org transsonic/subsonic flow substantially decelerated by thermal plasma within the inner $(\sim 1 \mathrm{kpc})$ region of the host giant elliptical galaxy (Komissarov 1994; Bowman et al. 1996; Bicknell 1995; Kaiser \& Alexander 1997);

- there are more fundamental differences between the two classes, involving the nature of the central black hole and the composition of jets, i.e. $\mathrm{e}^{-}-\mathrm{e}^{+}$plasma for FR I sources, while $\mathrm{e}^{-}-\mathrm{p}$ could be preferred in the case of FR II sources (Reynolds et al. 1996a,b; Meier et al. 1997; Meier 1999; Celotti et al. 1997);

- the differences in the jet power/thrust which, together with the properties of circumgalactic medium, would determine how soon the jets' collimation is disrupted (Gopal-Krishna \& Wiita 1988; Gopal-Krishna 1991; Gopal-Krishna et al. 1996).

Gopal-Krishna \& Wiita (2000) - hereafter G-KW - have introduced a new group of double radio sources called HYbrid MOrphology Radio Sources (HYMORS), where the two lobes exhibit clearly different FR morphological types. They argue that HYMORS could be used to constrain the theoretical models proposed for the FR-dichotomy, namely, that the existence of HYMORS supports the interpretation based upon jet interaction with the external medium or - at least - that the models based upon the differences in the nature of central massive black holes or jet compositions do not seem to be viable, since it is difficult to assume a physical process in which the same central engine could produce two different types of jets.

The six HYMORS in the sample established by G-KW were selected as a result of a literature search. Thus, their 
Table 1. Positions of the centres of subareas searched for HYMORS.

\begin{tabular}{ccc}
\hline \hline \multirow{2}{*}{ Subarea } & \multicolumn{2}{c}{ Position of the centre $(\mathrm{J} 2000)$} \\
\cline { 2 - 3 } & RA & Dec \\
\hline 1 & $10^{\mathrm{h}} 00^{\mathrm{m}}$ & $15^{\circ} 00^{\prime}$ \\
2 & $10^{\mathrm{h}} 30^{\mathrm{m}}$ & $30^{\circ} 00^{\prime}$ \\
3 & $11^{\mathrm{h}} 00^{\mathrm{m}}$ & $45^{\circ} 00^{\prime}$ \\
4 & $12^{\mathrm{h}} 30^{\mathrm{m}}$ & $50^{\circ} 00^{\prime}$ \\
5 & $13^{\mathrm{h}} 30^{\mathrm{m}}$ & $35^{\circ} 00^{\prime}$ \\
\hline
\end{tabular}

sample is not homogeneous in many respects. To circumvent this problem, a programme to find HYMORS candidates in one comprehensive survey using a single selection procedure was undertaken. This procedure, the observations, and the data reduction process are described in Sect. 2. The properties of three sources that eventually turned out to fulfill the criteria of the HYMORS class and two others that are likely to fall into this category are covered in Sect. 3 and further discussed in Sect. 4.

\section{Sample selection, observations, and data reduction}

The high sensitivity and the resolution of the VLA Faint Images of the Radio Sky at Twenty-centimeters (FIRST) survey (White et al. 1997) ${ }^{1}$ offers a unique possibility of studying the morphologies of a large number of moderately weak radio galaxies, so it is also a suitable database for pursuing the search for HYMORS. A high galactic latitude area limited by $\mathrm{RA}=\left\langle 11^{\mathrm{h}}-14^{\mathrm{h}}\right\rangle$ and Dec $=\left\langle 15^{\circ}-50^{\circ}\right\rangle$ was inspected with the aim of finding sources that might belong to the HYMORS class. Our survey did not completely cover the area indicated above, but instead five subareas randomly located inside that region were chosen. Each subarea has a radius of $8^{\circ} 20^{\prime}\left(30000^{\prime \prime}\right)$. The positions of the centres of these subareas are given in Table 1.

As a first step, all sources with flux densities $F_{1.4 \mathrm{GHz}} \geq$ $20 \mathrm{mJy}$ and angular size $\theta>8^{\prime \prime}$ were selected. Those values were chosen because we preferred to search for relatively bright extended sources. Maps of more than 1700 sources extracted from FIRST were inspected visually and the candidates selected. As a result, a sample consisting of 21 sources that appear to be HYMORS-like in the FIRST images was established.

This initial candidate sample was observed on 11 Nov. 2003 with the VLA in B-conf. at $4.9 \mathrm{GHz}$, which yields a resolution of $\sim 1$ ". 4 . Each programme source was observed for $5.5 \mathrm{~min}$. 3C 286 was used as a flux density calibrator, and sources selected from the List of the VLA calibrators nearby to target objects were used as their respective phase calibrators. The sources were grouped into a few subsamples according the positions of the targets on the sky, and one phase calibrator was used for each subsample. The data were reduced using the AIPS package. After the initial amplitude calibration, a few cycles of self-calibration were applied. For brighter sources, the

\footnotetext{
${ }^{1}$ Official website: http://sundog.stsci.edu
}

amplitude self-calibration was also carried out. The corrected data were further processed using IMAGR.

As a result of a careful inspection of 21 VLA 4.9-GHz images, 16 sources were rejected. The reasons for this are described in Appendix A. The most common reason is the lack of a well-defined component that terminates the FR II lobe and as such could be responsible for the edge brightening. In many such cases the lobes are generally diffuse and weak. Optionally, sources of this kind can be featured by a (relatively) strong core. If this is the case, the object is likely to have undergone re-ignition of the activity (Marecki et al. 2006). On the other hand, the lack of a dominating core, together with a "fuzzy" shape of the lobes, is a good signature of the cessation of activity. Sources possessing these features are sometimes termed "faders" and, although relatively rare, have been observed mostly in surveys of ultra-steep spectrum sources (Röttgering et al. 1994; De Breuck et al. 2000; Cohen et al. 2004). Six sources out of 16 rejects seem to be faders, and four others are likely to be restarted. As the images of these rejected sources might be considered interesting per se, we include them as Fig. A.1.

The remaining 5 out of 21 sources observed at $4.9 \mathrm{GHz}$ using the VLA appeared to be HYMORS according to the same criteria as those adopted by G-KW. The follow-up observations of these objects were carried out with the VLA in A-conf. at $1.4 \mathrm{GHz}$ on 20 and 21 Sep. 2004. Again, 3C 286 was used as the flux density calibrator, and programme sources were grouped so that a single phase calibrator could be used for each group. Each target source was observed for $\sim 30$ min divided into six $\sim 5$-min scans.

The basic parameters of the new HYMORS are given in Table 2, and the final images shown in Figs. 1 to 5. For comparison, their respective FIRST images are also included. The positions listed in Table 2 are those of the core components as seen in the 4.9-GHz VLA maps fitted using AIPS task JMFIT. JMFIT was also used to measure the flux densities of the other main components, and these measurements are shown in Table 3. For three sources (J1154+513, J1206+503 and $\mathrm{J} 1313+507$ ), spectral index maps were obtained from the two-frequency VLA images convolved with a common circular Gaussian beam $\left(11^{\prime \prime} 75 \times 11^{\prime \prime} .75\right)$ and are shown in Figs. 1 to 3. As all five sources investigated here are included in the Release 4 of the Sloan Digital Sky Survey (SDSS/DR4) ${ }^{2}$, their SDSS objID's are listed in Table 2 (Col. 2).

\section{Notes on individual sources}

J1154+513 (Fig. 1). In the FIRST image, this object (also known as $4 \mathrm{C}+51.28)$ appears as a triple. There is a hint that the south-eastern component, together with the central feature could form an unresolved FR I jet. This conjecture was fully confirmed by our VLA observations at $4.9 \mathrm{GHz}$ and $1.4 \mathrm{GHz}$. According to the images resulting from them, it is evident that the south-eastern part of the source has a classical FR I

2 The up-to-date version at the time of writing. 
Table 2. The newly discovered HYMORS. Positions are those of the core components.

\begin{tabular}{|c|c|c|c|c|c|c|c|c|}
\hline \multirow{3}{*}{$\begin{array}{c}\text { Source } \\
\text { name }\end{array}$} & \multirow{3}{*}{$\begin{array}{l}\text { SDSS } \\
\text { objID }\end{array}$} & \multirow[b]{2}{*}{ RA } & \multirow[b]{2}{*}{ Dec } & \multicolumn{5}{|c|}{ Flux density [mJy] } \\
\hline & & & & \multicolumn{3}{|c|}{$1.4 \mathrm{GHz}$} & \multicolumn{2}{|c|}{$4.9 \mathrm{GHz}$} \\
\hline & & \multicolumn{2}{|c|}{$(\mathrm{J} 2000)$} & FIRST & NVSS & this paper & GB6 & this paper \\
\hline $\mathrm{J} 1154+513$ & 587732134310838583 & $\begin{array}{lll}11 & 53 & 46.43\end{array}$ & $\begin{array}{lll}+51 & 17 & 04.1\end{array}$ & 495 & 483 & 490 & 137 & 131 \\
\hline $\mathrm{J} 1206+503$ & 587732483822518553 & $\begin{array}{lll}12 & 06 & 22.39\end{array}$ & $+50 \quad 1744.3$ & 241 & 170 & 265 & 75 & 88 \\
\hline $\mathrm{J} 1313+507$ & 588018054571360837 & $\begin{array}{lll}13 & 13 & 25.78\end{array}$ & +504206.2 & 277 & 252 & 230 & 84 & 86 \\
\hline $\mathrm{J} 1315+516$ & 588018055645102348 & $13 \quad 14 \quad 38.12$ & $+5134 \quad 13.4$ & 93 & 144 & 48 & 51 & 41 \\
\hline $\mathrm{J} 1348+286$ & 587739721369255946 & 134751.58 & +283629.6 & 241 & & & 117 & 105 \\
\hline
\end{tabular}
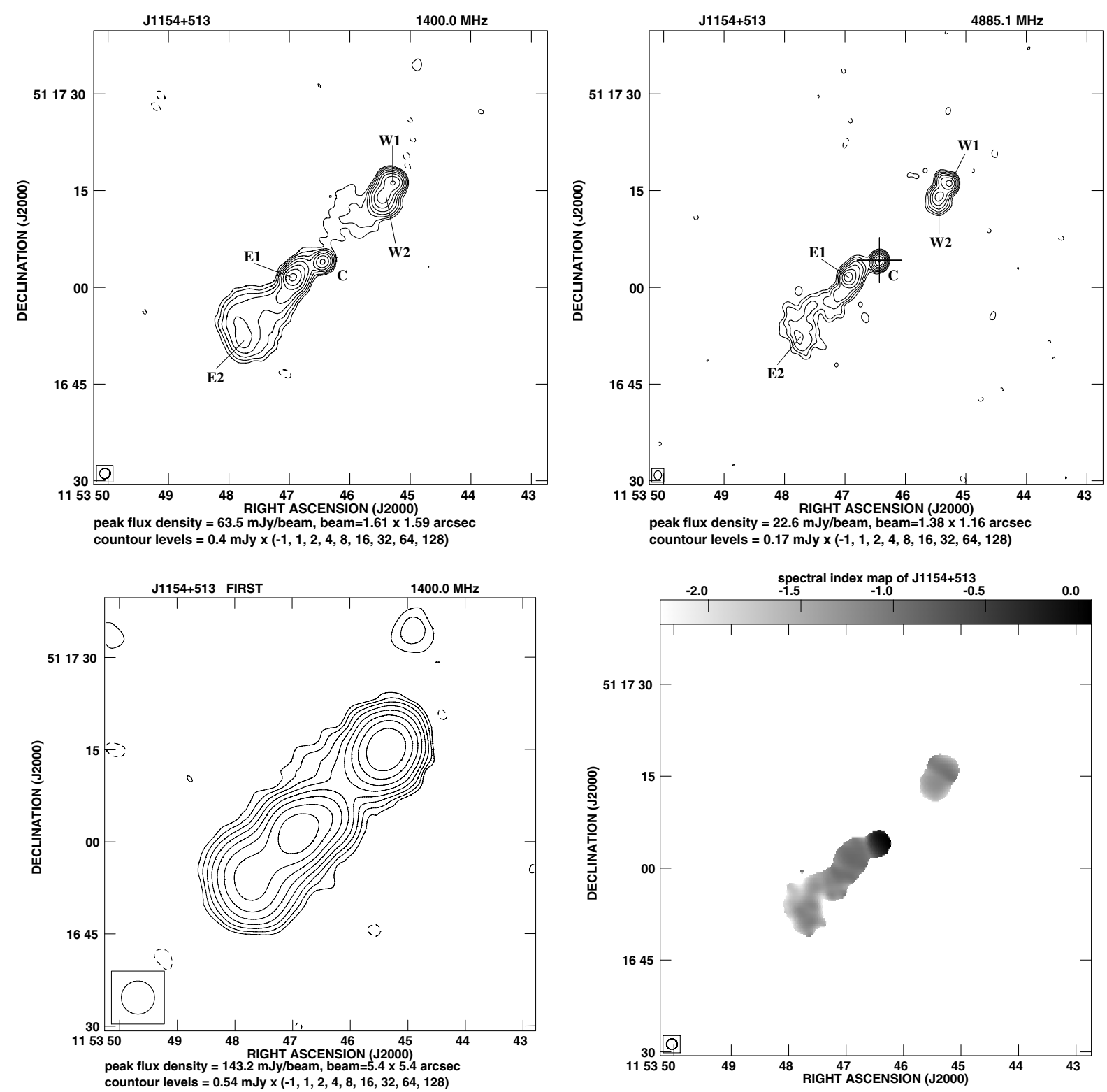

Fig. 1. VLA maps of J1154+513. Upper left: VLA in A-conf. at $1.4 \mathrm{GHz}$. Upper right: VLA in B-conf. at $4.9 \mathrm{GHz}$. The position of the optical object extracted from SDSS is marked with a cross. Lower left: FIRST map. Lower right: spectral index map obtained from the two-frequency VLA images shown in the upper panels.

structure with flat spectrum $(\alpha=-0.25)^{3}$ bright core "C" and a prominent hotspot "E1" placed inside the jet. On the other hand, the north-western part is a typical FR II lobe ("W2") with

\footnotetext{
${ }^{3}$ Throughout this paper $\alpha$ is defined as: $S \propto \nu^{\alpha}$.
}

a hotspot ("W1"). The "E2" component has a steeper spectrum $(\alpha=-1.45)$ than E1 $(\alpha=-1.05)$. Steepening of the spectrum with the increasing distance from the core is a wellknown feature of FR I radio galaxies (see e.g. Katz-Stone \& Rudnick 1997). The north-western lobe has a structure that is 

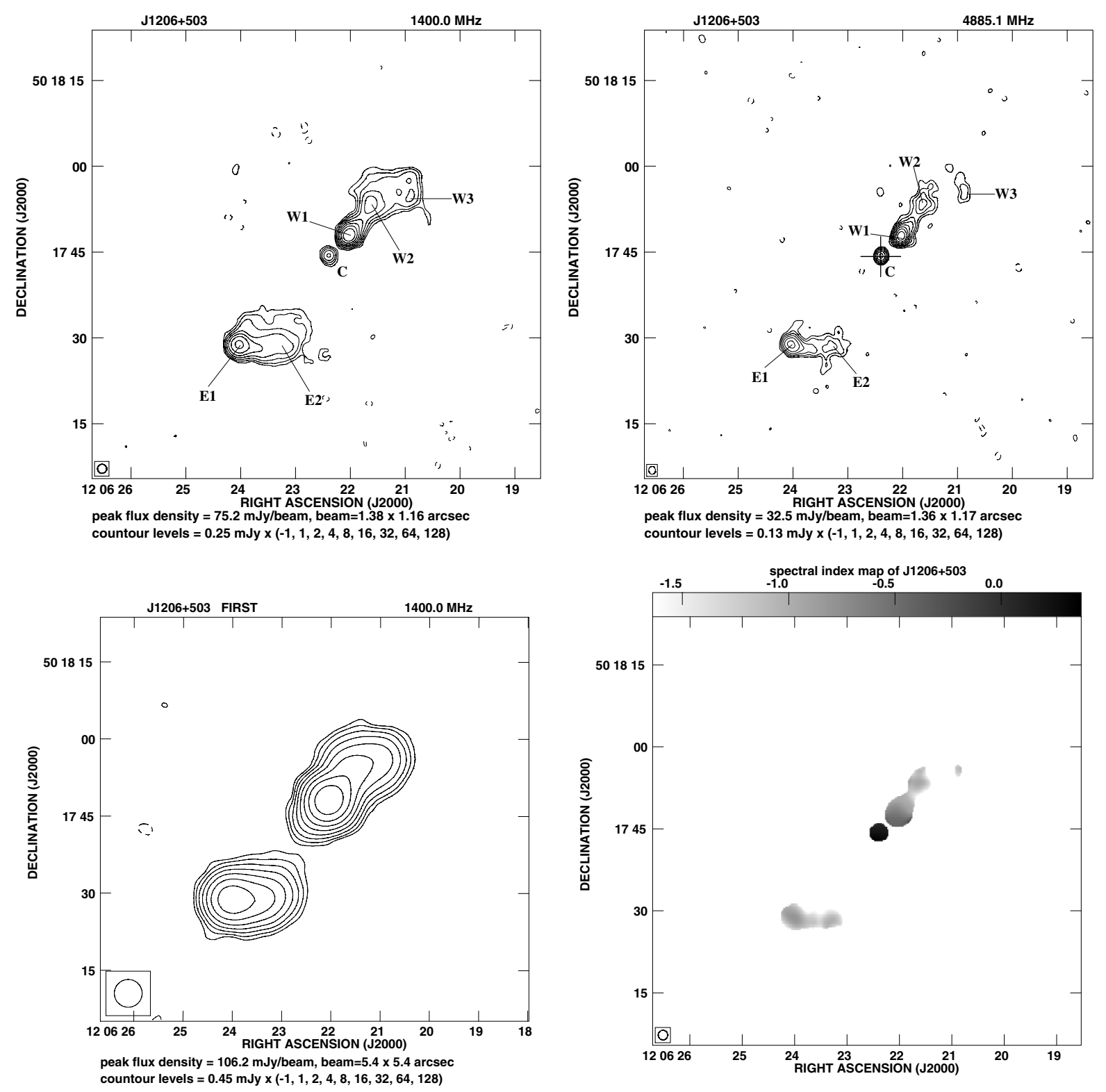

Fig. 2. VLA maps of $\mathbf{J 1 2 0 6 + 5 0 3}$. Upper left: VLA in A-conf. at 1.4 GHz. Upper right: VLA in B-conf. at $4.9 \mathrm{GHz}$. The position of the optical object extracted from SDSS is marked with a cross. Lower left: FIRST map. Lower right: spectral index map obtained from the two-frequency VLA images shown in the upper panels.

typical for FR II-type lobes with flattening of spectrum toward the hotspot (see e.g. Leahy et al. 1989). Therefore, both the morphology, as seen in the total intensity maps, and the spectral index map fully confirm a hypothesis that there are two different types of jets present in one object. It is also to be noted that there is a connection (bridge) between the northern FR II-type lobe and the core "C" clearly visible at $1.4 \mathrm{GHz}$. Given that it is not present in the $4.9-\mathrm{GHz}$ image, the bridge must have a very steep spectrum. This remains in full agreement with Leahy et al. (1989) leaving no room for doubt that the two parts of the source as seen in the 4.9-GHz VLA image are not a coincidence. Our measurements of the flux densities are in a very good agreement with GB6 $(4.85 \mathrm{GHz})$ and NVSS $(1.4 \mathrm{GHz})$ catalogues. This source is very similar to $1004+130$ and $4 \mathrm{C}-03.64$, both presented in G-KW, and as such is a perfect example of a HYMORS.
The $\mathrm{J} 1154+513$ radio source has an optical counterpart in SDSS/DR4, a galaxy with $m_{\mathrm{R}}=21.46$. There is a hint of irregularity in the shape of the host galaxy. The redshift of this object is unknown at present.

$\mathbf{J 1 2 0 6 + 5 0 3 ~ ( F i g . ~ 2 ) . ~ I n ~ t h e ~ F I R S T ~ i m a g e ~ t h i s ~ s o u r c e ~ h a s ~}$ two main components. The south-eastern one is an FR II-type lobe, whereas the north-western part of source has a barely resolved core-jet structure. The images resulting from our VLA follow-up observations confirm this. The north-western part consists of three conspicuous features, a flat spectrum core ("C") and the jet with two brighter knots ("W1" and "W2") and a diffuse "plume" ("W3"), which together make a clear case for a FR I-type jet, whereas the eastern lobe ("E2") with a hotspot ("E1") is an FR II-type lobe specimen. Thus, the morphology of J1206+503 is fully consistent with a HYMORS class definition. As in the case of J1154+513, the spectral index map of 

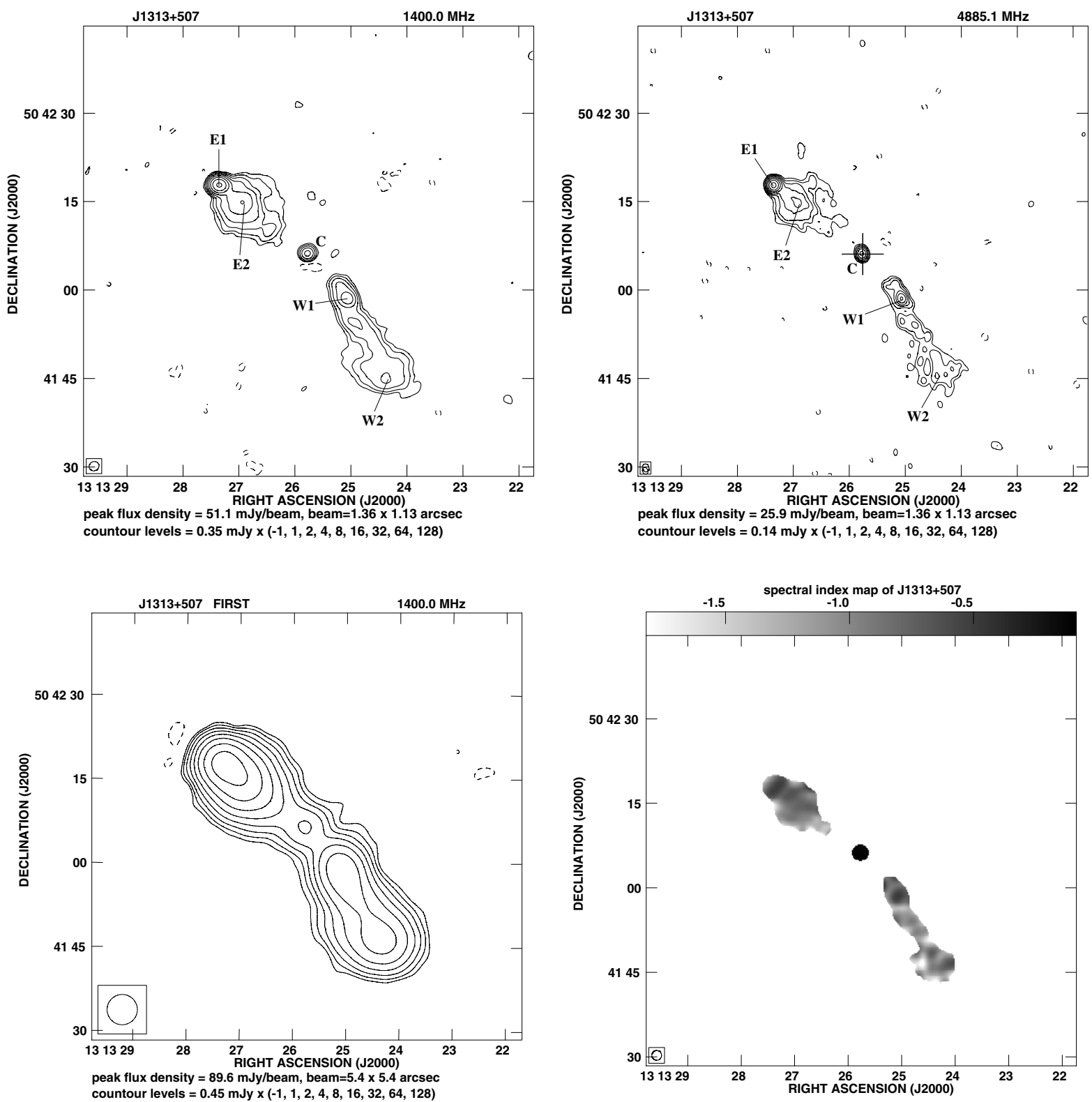

Fig. 3. VLA maps of $\mathbf{J 1 3 1 3 + 5 0 7}$. Upper left: VLA in A-conf. at $1.4 \mathrm{GHz}$. Upper right: VLA in B-conf. at $4.9 \mathrm{GHz}$. The position of the optical object extracted from SDSS is marked with a cross. Lower left: FIRST map. Lower right: spectral index map obtained from the two-frequency VLA images shown in the upper panels.

$\mathrm{J} 1206+503$ confirms that there are two different types of radio jets.

This source has an $m_{\mathrm{R}}=20.86$ optical counterpart in SDSS/DR4. The host galaxy seems to be irregular and has two close companion galaxies: SDSS J120622.11+501740.9 $\left(m_{\mathrm{R}}=22.66\right)$ and SDSS J120621.94+501743.2 $\left(m_{\mathrm{R}}=21.21\right)$ located 4". 3 and 4.' 6 off the position of the optical object identified with the core of the HYMORS, respectively. The redshifts of all three objects are not known at present.

$\mathbf{J 1 3 1 3 + 5 0 7}$ (Fig. 3). This source consists of four main components in the FIRST image. A well-developed north-eastern FR II structure, a putative core with an optical counterpart $m_{\mathrm{R}}=20.90$ in SDSS/DR4 - and a south-western structure that could be an unresolved FR I jet. Our new VLA images confirm that the latter is actually FR I-type. With the "E1" component being the hotspot of an FR II-type lobe and "W2" FR I-type plume, $\mathrm{J} 1313+507$ is - again - a very good example of a HYMORS, quite similar to $4 \mathrm{C}-03.64(\mathrm{G}-\mathrm{KW})$. The redshift of this object is unknown at present.

J1315+516 (Fig. 4). The FIRST image strongly suggests that this source has a HYMORS morphology. In the 4.9-GHz image, the eastern (apparently FRI) jet is not reproduced well, which means it has a diffuse structure without any welllocalised features except the region denoted as "E1". The western part consists of a hotspot ("W1") inside a well-defined FR II-like lobe. Given that a substantial amount of flux is missing when observing the source at $1.4 \mathrm{GHz}$ in B-conf. (FIRST) and A-conf. (our observations), a significant diffuse component must exist that is not reproduced in either our $1.4-\mathrm{GHz}$ image or the FIRST map. Also, the weak radio structures in this 

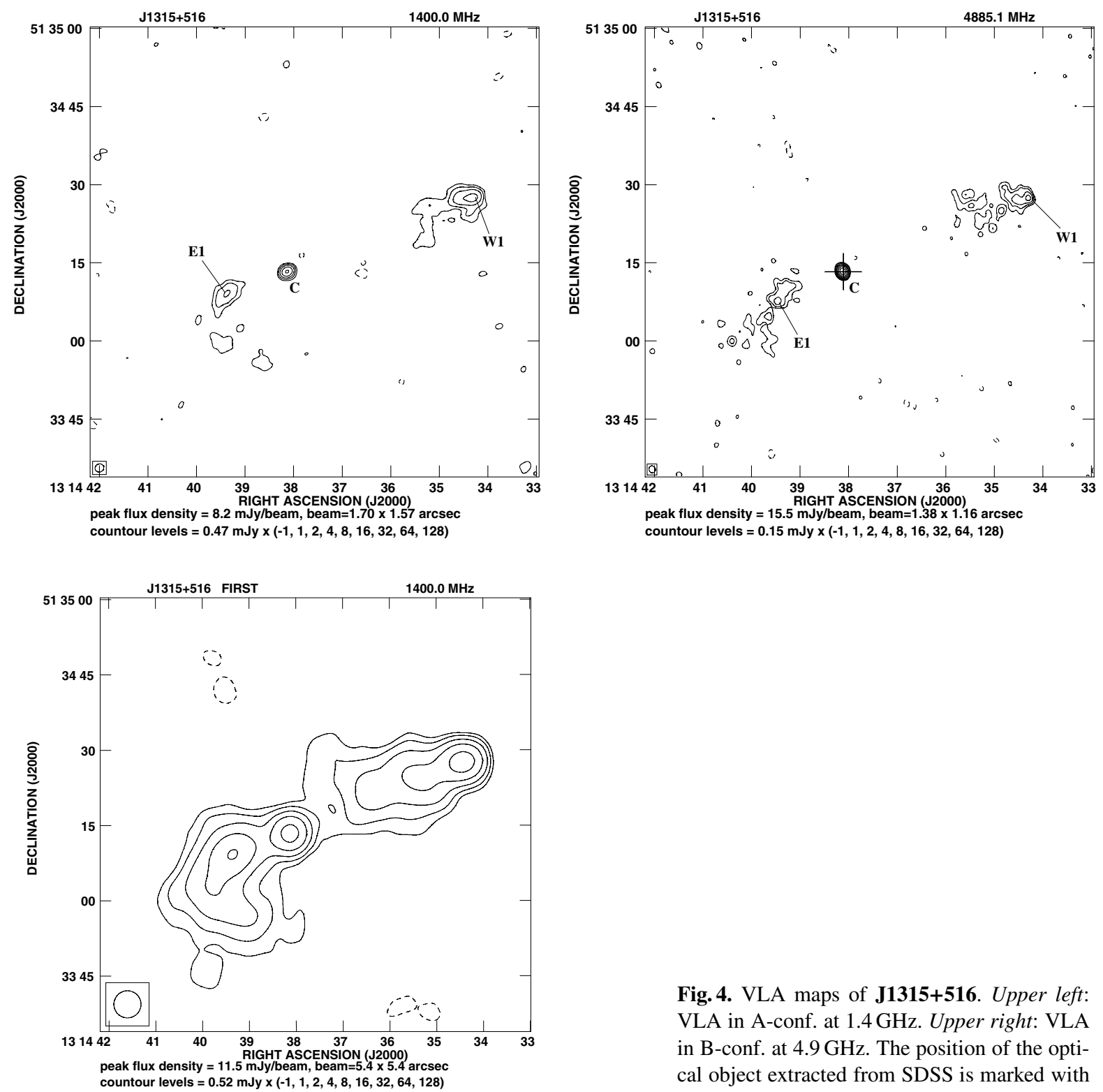

Fig. 4. VLA maps of J1315+516. Upper left: VLA in A-conf. at $1.4 \mathrm{GHz}$. Upper right: VLA in B-conf. at $4.9 \mathrm{GHz}$. The position of the optical object extracted from SDSS is marked with a cross. Lower: FIRST map.

object do not allow a good map of the spectral index to be made. The fact that the source is strongly core-dominated, whereas the lobes are very diffuse, is an indication that it could be a restarted source - see Sect. 4 for further discussion.

The core of $\mathrm{J} 1315+516$ has an optical counterpart of $m_{\mathrm{R}}=19.77$, and there are two close companion galaxies: SDSS J131437.79+513410.6 $\left(m_{\mathrm{R}}=22.76\right)$ and SDSS J131437.10+513408.1 $\left(m_{\mathrm{R}}=20.70\right)$ located $4 . \prime 1$ and $10^{\prime \prime} 8$ off the position of the optical object identified with the core of the HYMORS. The redshifts of all these three objects are not known at present.

$\mathbf{J 1 3 4 8 + 2 8 6}$ (Fig. 5). The structure of this object is somewhat similar to that of J1315+516: a hotspot of the FR IIlike lobe ("W1"), and a fuzzy, possible FR I jet ("E1") can be recognised. Due to the bad quality of the observational data, it was not possible to make a good VLA image of this source at $1.4 \mathrm{GHz}$, so the FR I nature of the "E1" structure is not certain, and this is the least convincing HYMORS among the five shown here.

$\mathrm{J} 1348+286$ is associated with an X-ray object RXJ1347.7+2836. According to SDSS/DR4 this object is a QSO $\left(m_{\mathrm{R}}=17.27\right)$ at the redshift of $z=0.7407$ (Muñoz et al. 2003). (A $m_{\mathrm{R}}=14.5$ field star is within 2 '.7 the QSO.) This is the farthest HYMORS known up to date. At this redshift and given that the total flux of the source extracted from FIRST is $241 \mathrm{mJy}$, the logarithm of the monochromatic luminosity at $1.4 \mathrm{GHz}$ amounts to 26.5, assuming $H_{0}=75 \mathrm{~km} \mathrm{~s}^{-1} \mathrm{Mpc}^{-1}$ and $q_{0}=0.5$ in accordance with the data in Table 1 in G-KW. It looks, therefore, that this distant HYMORS is an order of magnitude more powerful than the nearby ones examined by G-KW $\left(\log L_{\mathrm{R}}=25.4\right.$ for three of them), although it must be borne in mind that for $1004+130 \log L_{\mathrm{R}}=26.3$, which is quite close to the respective value for $\mathrm{J} 1348+286$. 

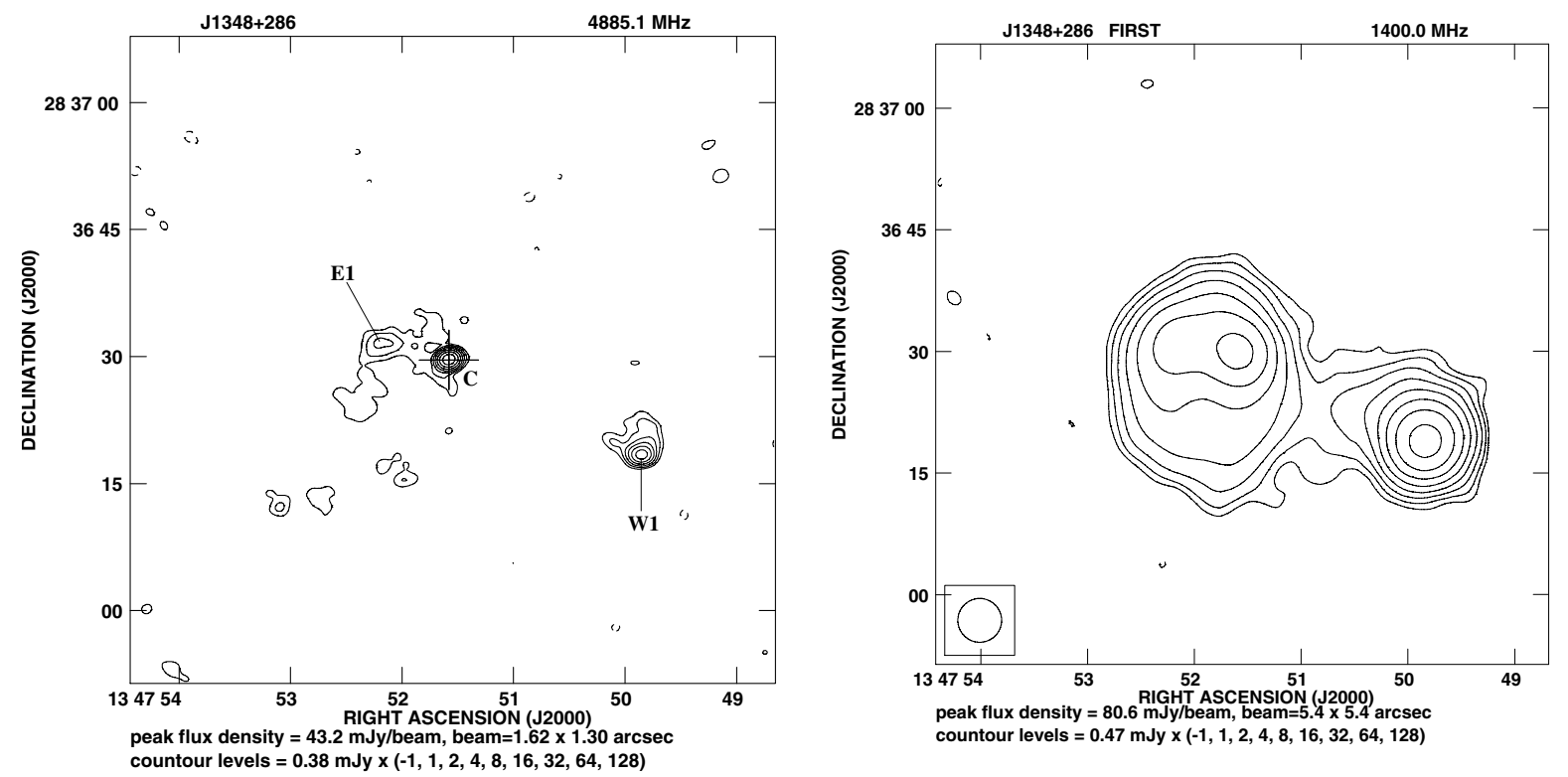

Fig. 5. VLA maps of $\mathbf{J 1 3 4 8 + 2 8 6}$. Left: VLA in B-conf. at $4.9 \mathrm{GHz}$. The position of the optical object extracted from SDSS is marked with a cross. Right: FIRST map.

Table 3. The flux densities and spectral index of main components.

\begin{tabular}{llrrr}
\hline \hline Source & Com- & \multicolumn{2}{c}{ Flux density $[\mathrm{mJy}]$} & Spectral \\
\cline { 3 - 4 } & ponent & $1.4 \mathrm{GHz}$ & $4.9 \mathrm{GHz}$ & index \\
\hline J1154+513 & C & 33.4 & 24.7 & -0.25 \\
& E1 & 107.5 & 29.3 & -1.05 \\
& E2 & 54.0 & 9.1 & -1.45 \\
& W1 & 100.9 & 22.0 & -1.24 \\
& W2 & 99.4 & 18.6 & -1.36 \\
\hline J1206+503 & C & 4.5 & 5.8 & 0.21 \\
& E1 & 52.1 & 15.5 & -0.99 \\
& E2 & 30.9 & 4.6 & -1.54 \\
& W1 & 105.9 & 45.9 & -0.68 \\
& W2 & 26.5 & 5.2 & -1.32 \\
& W3 & 9.4 & 1.4 & -1.56 \\
\hline J1313+507 & C & 7.0 & 6.1 & -0.11 \\
& E1 & 69.8 & 30.8 & -0.66 \\
& E2 & 58.0 & 15.3 & -1.08 \\
& W1 & 17.9 & 6.7 & -0.80 \\
\hline J1315+516 & C & 16.0 & 13.1 & 0.09 \\
& E1 & 3.1 & 21.7 & -0.55 \\
& W1 & 3.6 & 19.9 & -0.58 \\
\hline J1348+286 & C & & 44.7 & \\
& E1 & & 8.7 & \\
& W1 & & 24.7 & \\
\hline
\end{tabular}

\section{Discussion}

HYMORS could serve as a discriminator between a range of theories used to explain the origin of the FR-dichotomy. As pointed out by G-KW, the existence of HYMORS does not favour the models based upon fundamental differences between the central engines, such as black hole spin or jet composition. It seems that in the case of HYMORS some type of jetmedium interaction on the scale of kiloparsecs may play a crucial role and has a significant impact on the FR-dichotomy.
Thus, if it is assumed that the properties of the intracluster or intergalactic medium are an important factor in the evolution of radio sources, then the phenomenon of HYMORS might be explained. It is likely that, during the evolution of a cluster of galaxies, there are many interactions between the members of the cluster. If the interactions are very frequent and strong, it may be expected that the properties of the intracluster medium (density, temperature, pressure) in the cluster could vary in time and space. Assuming that there is a difference of the properties in the medium between the opposite sides of a host galaxy, a HYMORS might emerge even if the jets are identical when launched from the central engine. A recent study by Cao \& Rawlings (2004) also provides support for the hypothesis that there is no fundamental difference between FR I and FR II engines.

It is to be noted that three sources out of the five investigated in detail - $\mathrm{J} 1154+513, \mathrm{~J} 1206+503$ and $\mathrm{J} 1313+507$ - are not core-dominated (see Table 3), and their structures are imaged very well using the VLA with 1 .' 4 resolution, whereas the remaining two $-\mathrm{J} 1315+516$ and $\mathrm{J} 1348+286$ - are clearly coredominated, but their jets, particularly those at the "FR I side", are fuzzy and not reproduced well in our maps, as substantial amounts of the flux are missing. We suggest that these two objects, apart from being likely examples of HYMORS, can also be labeled as "core-dominated triples". Radio sources of this sort extracted from FIRST in a systematic manner have been investigated by Marecki et al. (2006). They claim that such objects are restarted and that the dominance of their cores is a direct consequence of being re-oriented in the course of a merger event. As a result of this, they are now more beamed towards the observers whereas the lobes are not fuelled any longer, so they have entered the so-called "coasting" phase of their evolution. However, if these two are not restarted sources, then the core prominence would imply that both lobes are greatly 
foreshortened by projection, in which case the apparent FR I structure could be an FR II-type lobe seen nearly head-on.

\section{Summary}

The main objective of this work was to expand the number of known HYMORS by means of a systematic study of images resulting from a high sensitivity radio sky survey. A sample of more than 1700 sources from the FIRST catalogue was examined and 21 candidates selected. After re-observation with the VLA in B-conf. at $4.9 \mathrm{GHz}$, three sources turned out to be certain HYMORS and two others are very likely to fall into this rare category. Our "success rate" therefore is somewhat lower than that of G-KW, as they found 6 HYMORS among the somewhat more than 1000 objects they examined from a search of the literature. It must be stressed, however, that in the case of the FIRST catalogue, the resolution of the images is often worse than those in the images selected by G-KW. As a result we might well have rejected some true HYMORS during the initial selection process. Follow-up VLA observations in A-conf. at $1.4 \mathrm{GHz}$ provided a confirmation that those three objects labelled as HYMORS based upon the inspection of 4.9-GHz images indeed fulfill the criteria of this class. They also made the preparation of spectral index maps possible. The spectral index gradients additionally support the identifications of the FR I and FR II sides. As a result, the conjecture that HYMORS indeed contain two different types of radio jets has gained strong support.

Acknowledgements. The VLA is operated by the U.S. National Radio Astronomy Observatory, which is operated by Associated Universities, Inc., under cooperative agreement with the National Science Foundation.

This research has made use of the NASA/IPAC Extragalactic Database (NED), which is operated by the Jet Propulsion Laboratory, California Institute of Technology, under contract with the National Aeronautics and Space Administration.

Use has been made of the fourth release of the Sloan Digital Sky Survey (SDSS) Archive. Funding for the creation and distribution of the SDSS Archive has been provided by the Alfred P. Sloan Foundation, the Participating Institutions, the National Aeronautics and Space Administration, the National Science Foundation, the U.S. Department of Energy, the Japanese Monbukagakusho, and the Max Planck Society. The SDSS Web site is http://www.sdss.org/. The SDSS is managed by the Astrophysical Research Consortium (ARC) for the Participating Institutions. The Participating Institutions are The University of Chicago, Fermilab, the Institute for Advanced Study, the Japan Participation Group, The Johns Hopkins University, Los Alamos National Laboratory, the Max-Planck-Institute for
Astronomy (MPIA), the Max-Planck-Institute for Astrophysics (MPA), New Mexico State University, University of Pittsburgh, Princeton University, the United States Naval Observatory, and the University of Washington.

We are very grateful to Prof. Paul Wiita and Prof. Gopal-Krishna for carefully reading the manuscript and making a number of suggestions and corrections that led to a significant improvement in this paper.

\section{References}

Bicknell, G. V. 1995, ApJS, 101, 29

Bowman, M., Leahy, J. P., \& Komissarov, S. S. 1996, MNRAS, 279, 899

Cao, X., \& Rawlings, S. 2004, MNRAS, 349, 1419

Celotti, A., Padovani, P., \& Ghisellini, G. 1997, MNRAS, 286, 415

Cohen, A. S., Röttgering, H. J. A., Jarvis, M. J., Kassim, N. E., \& Lazio, T. J. W. 2004, ApJS, 150, 417

De Breuck, C., van Breugel, W., Röttgering, H. J. A., \& Miley, G. 2000, A\&AS, 143, 303

Fanaroff, B. L., \& Riley, J. M. 1974, MNRAS, 167, 31P

Giroletti, M., Giovannini, G., \& Taylor, G. B. 2005, A\&A, 441, 89

Gopal-Krishna 1991, A\&A, 248, 415

Gopal-Krishna, \& Wiita, P. J. 1988, Nature, 333, 49

Gopal-Krishna, \& Wiita, P. J. 2000, A\&A, 363, 507 (G-KW)

Gopal-Krishna, Wiita, P. J., \& Hooda, J. S. 1996, A\&A, 316, L13

Hine, R. G., \& Longair, M. S. 1979, MNRAS, 188, 111

Kaiser, C. R., \& Alexander, P. 1997, MNRAS, 286, 215

Katz-Stone, D. M., \& Rudnick, L. 1997, ApJ, 488, 146

Komissarov, S. S. 1994, MNRAS, 269, 394

Kunert-Bajraszewska, M., Marecki, A., Thomasson, P., \& Spencer, R. E. 2005, A\&A, 440, 93

Leahy, J. P., Muxlow, T. W. B., \& Stephens, P. W. 1989, MNRAS, 239, 401

Marecki, A., Thomasson, P., Mack, K.-H., \& Kunert-Bajraszewska, M. 2006, A\&A, in press [arXiv:astro-ph/0509497]

Meier, D. L., Edgington, S., Godon, P., Payne, D. G., \& Lind, K. R. 1997, Nature, 388, 350

Meier, D. L. 1999, ApJ, 522, 753

Muñoz, J. A., Falco, E. E., Kochanek, C. S., Lehár, J., \& Mediavilla, E. 2003, ApJ, 594, 684

Reynolds, C. S., di Matteo, T., Fabian, A. C., Hwang, U., \& Canizares, C. R. 1996a, MNRAS, 283, L111

Reynolds, C. S., Fabian, A. C., Celotti, A., \& Rees, M. J. 1996b, MNRAS, 283, 873

Röttgering, H. J. A., Lacy, M., Miley, G. K., Chambers, K. C., \& Saunders, R. 1994, A\&AS, 108, 79

Wall, J. V. 1980, Royal Society of London Philosophical Transactions Series A, 296, 367

White, R. L., Becker, R. H., Helfand, D. J., \& Gregg, M. D. 1997, ApJ, 475,479 
M. P. Gawroński et al.: Hybrid morphology radio sources from the FIRST survey, Online Material p 1

\section{Online Material}




\section{Appendix A}

Here we present notes on 16 sources selected from FIRST as possible candidates for HYMORS, observed at $4.9 \mathrm{GHz}$ with the VLA and rejected based on the results of these observations. The 4.9-GHz VLA images of these sources are shown in Fig. A.1 along with their respective cutouts from FIRST. The positions of the optical counterparts, if available in SDSS/DR4, are marked with crosses in the 4.9-GHz VLA images. They are also listed in Table A.1.

J1011+328. No compact feature that might be labelled as a hotspot seen in the VLA 4.9-GHz image, so there is no clear case of the FR II part. This object is a member of a cluster. The SDSS object 587739157655453889 is a possible core of this radio source, so that it is a Wide-Angle Tail (WAT) source. Another SDSS object (587739157655453835) is also within the boundary of the radio image presented, and it is marked with $\times$. J1011 +328 is possibly a fader.

$\mathbf{J 1 0 2 0 + 1 4 1}$. Although there is a hint in the VLA 4.9-GHz image that this could be a HYMORS, we label it as an FRI radio galaxy with a distorted structure. This galaxy at a redshift $z=0.146$ is a member of a cluster and has three close companions.

$\mathbf{J 1 0 2 1 + 4 4 4 . ~ N o ~ F R ~ I ~ p a r t ~ i s ~ s e e n ~ i n ~ t h e ~ V L A ~ 4 . 9 - G H z ~ i m - ~}$ age.

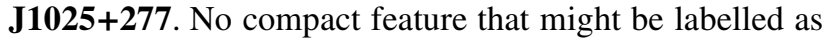
a hotspot is seen in the VLA $4.9-\mathrm{GHz}$ image so there is no clear case of the FR II part. There is a lack of visible jets and hotspots, and the radio core is weak $\left(S_{\text {core }} / S_{\text {tot }}=0.052\right)$. We suggest this source could be the same type as a fading galaxy $1855+37$ shown by Giroletti et al. (2005).

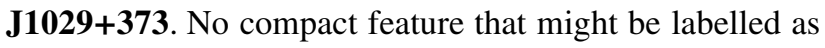
hotspot is seen in the VLA 4.9-GHz image so there is no clear case of the FR II part. As this source is core-dominated, it is likely to be restarted.

$\mathbf{J 1 0 3 0 + 2 9 9}$. The radio structure shows no indication of hotspots in the faint lobes or the radio core. This object resembles $1542+323$, the fading source presented by Kunert-Bajraszewska et al. (2005).

$\mathbf{J 1 0 4 5 + 5 2 3}$. This source is a possible fader.

$\mathbf{J 1 0 4 8 + 1 5 3}$. We failed to make a good 4.9-GHz image. Nevertheless, based on the FIRST image alone, this source looks like a very good HYMORS candidate.

$\mathbf{J 1 1 2 5 + 3 7 4}$. As this source is core dominated, it is likely to be restarted.

$\mathbf{J 1 2 0 3 + 5 3 8}$. The core is weak $\left(S_{\text {core }} / S_{\text {tot }}=0.067\right)$, and there is a lack of visible jets and hotspots. We suggest this source could be the same type as $1855+37$, the fading galaxy shown by Giroletti et al. (2005).

$\mathbf{J 1 2 0 7 + 4 8 3}$. No compact feature that might be labelled as a hotspot is seen in the VLA 4.9-GHz image, so although this source has a perfect northern FR I structure, there is no clear case of the FR II part.

$\mathbf{J 1 2 1 0 + 4 6 6 . ~ A s ~ t h i s ~ s o u r c e ~ i s ~ c o r e - d o m i n a t e d , ~ i t ~ i s ~ l i k e l y ~ t o ~}$ be restarted.

$\mathbf{J 1 3 0 3 + 3 1 8}$. No compact feature that might be labelled as a hotspot is seen in the VLA 4.9-GHz image, so there is no clear
Table A.1. Rejected HYMORS candidates with optical counterparts in SDSS/DR4. Positions are those of the optical object.

\begin{tabular}{|c|c|c|c|}
\hline \multirow{2}{*}{$\begin{array}{c}\text { Source } \\
\text { name }\end{array}$} & \multirow{2}{*}{$\begin{array}{l}\text { SDSS } \\
\text { objID }\end{array}$} & RA & Dec \\
\hline & & \multicolumn{2}{|c|}{$(\mathrm{J} 2000)$} \\
\hline $\mathrm{J} 1011+328$ & 587739157655453889 & 101106.12 & 324817.2 \\
\hline $\mathrm{J} 1020+141$ & 587735349636169918 & 101932.33 & 140301.8 \\
\hline $\mathrm{J} 1021+444$ & 588017112629313672 & 102116.44 & 442548.1 \\
\hline $\mathrm{J} 1029+373$ & 587738948284842142 & 102830.52 & 371510.7 \\
\hline $\mathrm{J} 1045+523$ & 587733080269914893 & 104438.63 & 521537.4 \\
\hline $\mathrm{J} 1125+374$ & 587739098595328059 & 112438.16 & 372240.3 \\
\hline $\mathrm{J} 1203+538$ & 587733079201349782 & 120302.05 & 534918.7 \\
\hline $\mathrm{J} 1207+483$ & 588297865268559953 & 120656.81 & 481512.5 \\
\hline $\mathrm{J} 1210+466$ & 588297863121273573 & 120947.09 & 463705.1 \\
\hline $\mathrm{J} 1303+318$ & 587739505547673753 & 130317.11 & 315002.6 \\
\hline $\mathrm{J} 1324+376$ & 587739099142553704 & 132412.40 & 373333.8 \\
\hline $\mathrm{J} 1339+394$ & 587738575145861682 & 133908.54 & 392624.1 \\
\hline $\mathrm{J} 1351+309$ & 587739609709674629 & 135103.40 & 305404.2 \\
\hline
\end{tabular}

case of the FR II part. It is a member of the Abell 1667 cluster. As this source is core-dominated, it is likely to be restarted.

$\mathbf{J 1 3 2 4 + 3 7 6}$. In the 4.9-GHz VLA image, the radio structure of this source consists of a core and diffuse lobes without hotspots, so that it is impossible to classify this source either as FR I or FR II. It is a member of the $\mathrm{ZwCl} \mathrm{1322.0+3750} \mathrm{cluster.}$ $\mathrm{J} 1324+376$ is a possible fader.

J1339+394. FRII radio galaxy with a bright core.

$\mathbf{J 1 3 5 1 + 3 0 9}$. FRI radio galaxy belonging to the $\mathrm{ZwCl} 1348.7+3109$ cluster. 
M. P. Gawroński et al.: Hybrid morphology radio sources from the FIRST survey, Online Material p 3
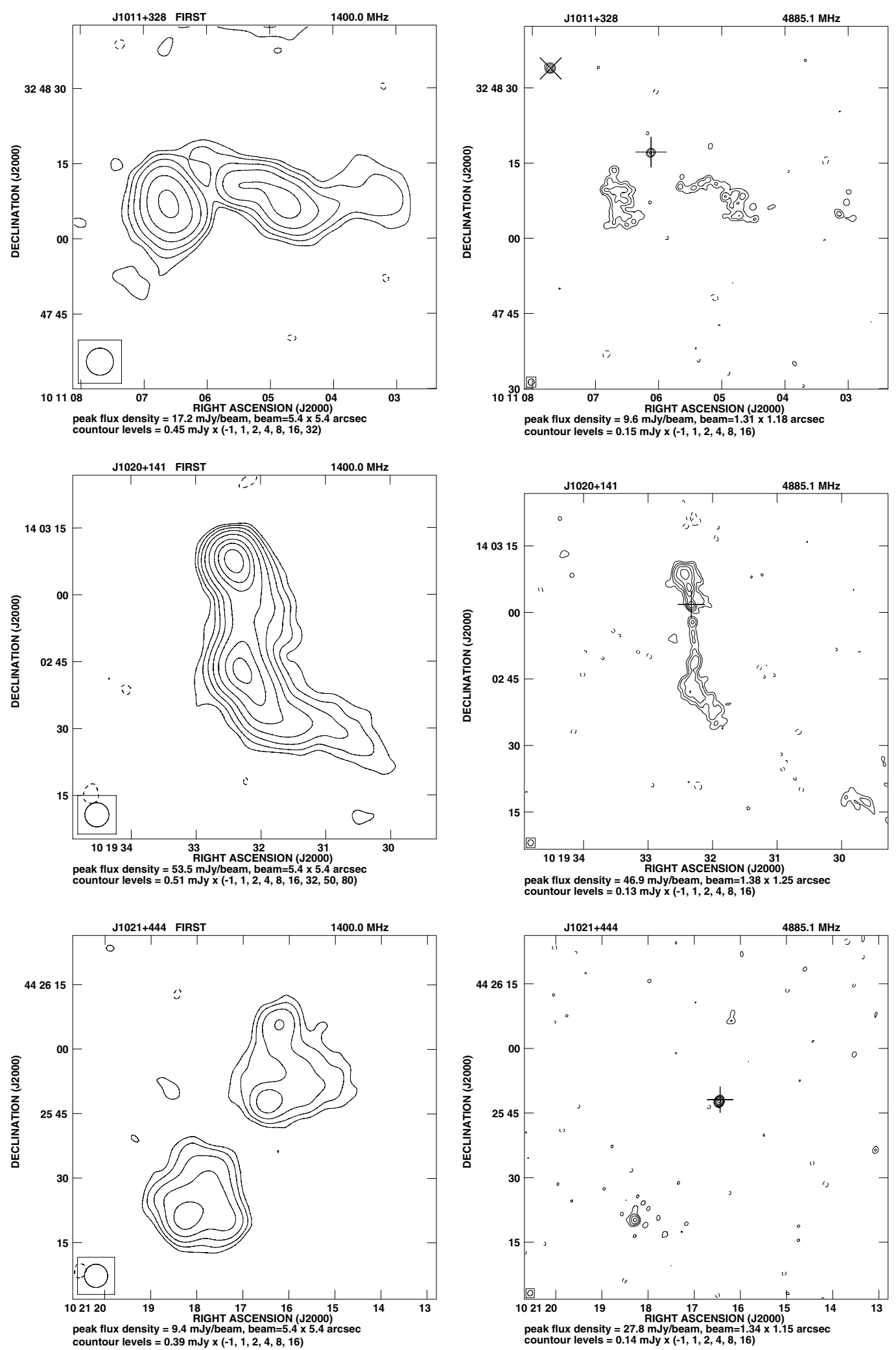

Fig. A.1. Sources rejected as HYMORS candidates. Left panels: FIRST maps. Right panels: 4.9 GHz VLA B-conf. maps from this study. 
M. P. Gawroński et al.: Hybrid morphology radio sources from the FIRST survey, Online Material p 4
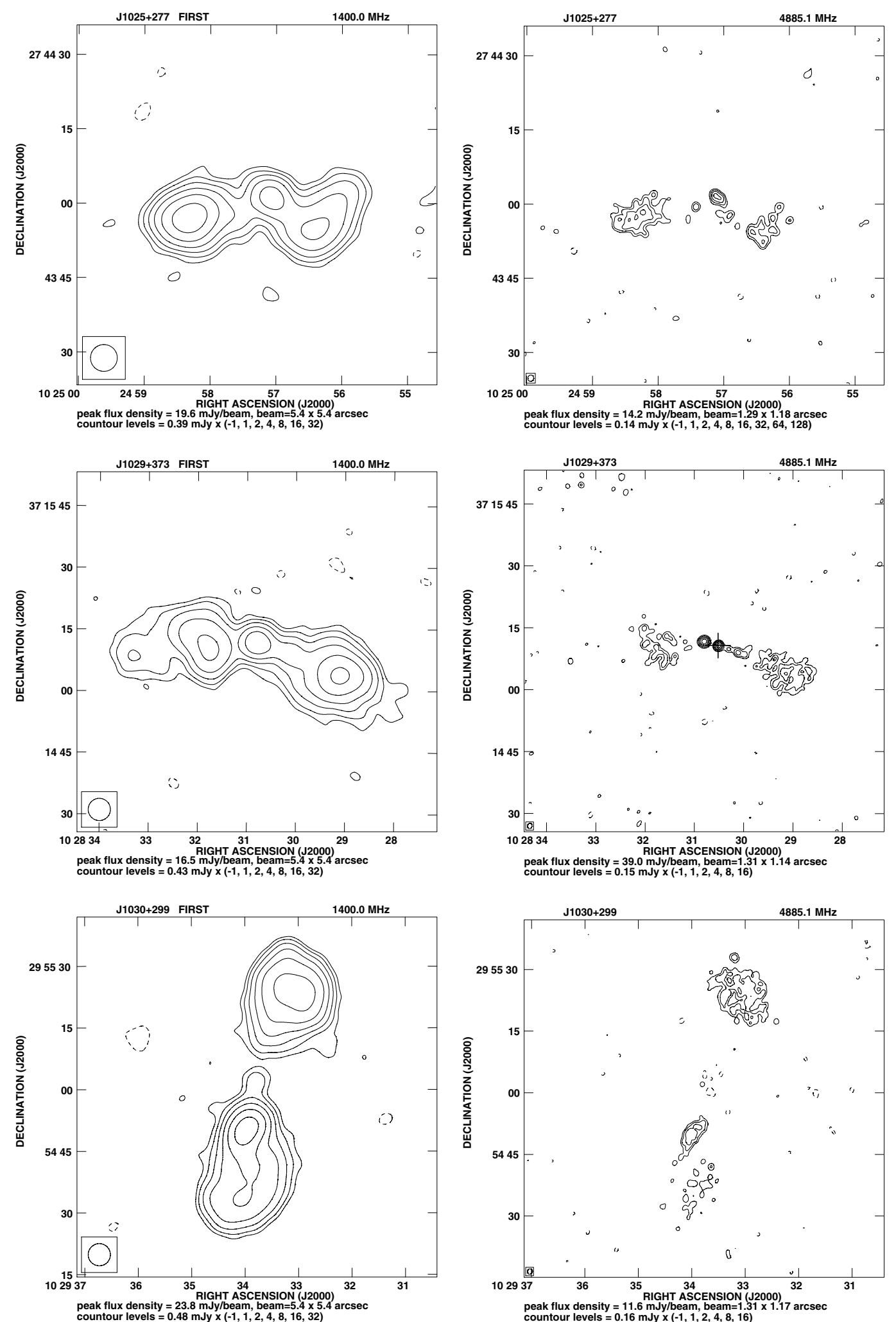

Fig. A.1. continued. 
M. P. Gawroński et al.: Hybrid morphology radio sources from the FIRST survey, Online Material p 5
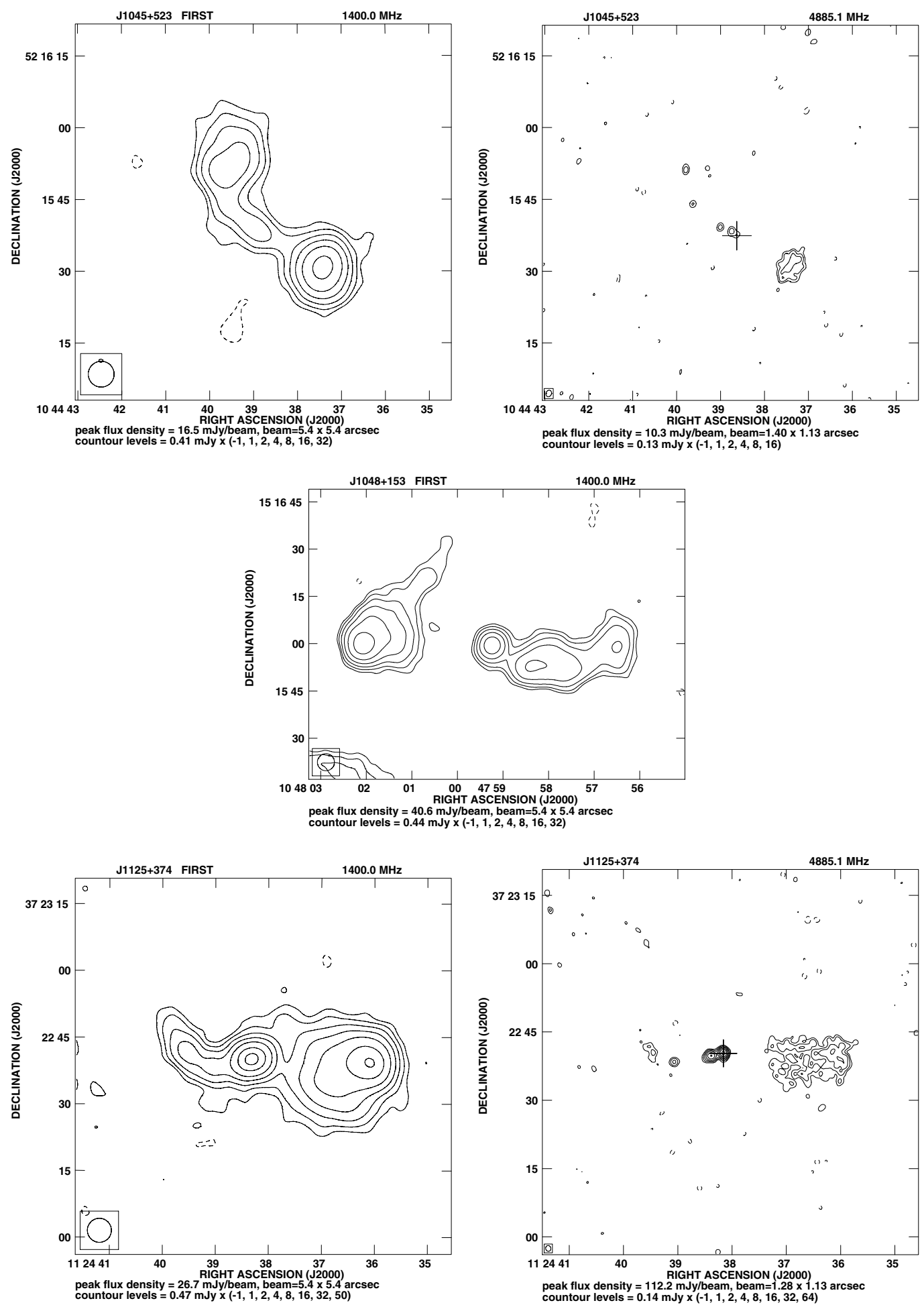

Fig. A.1. continued. 
M. P. Gawroński et al.: Hybrid morphology radio sources from the FIRST survey, Online Material p 6
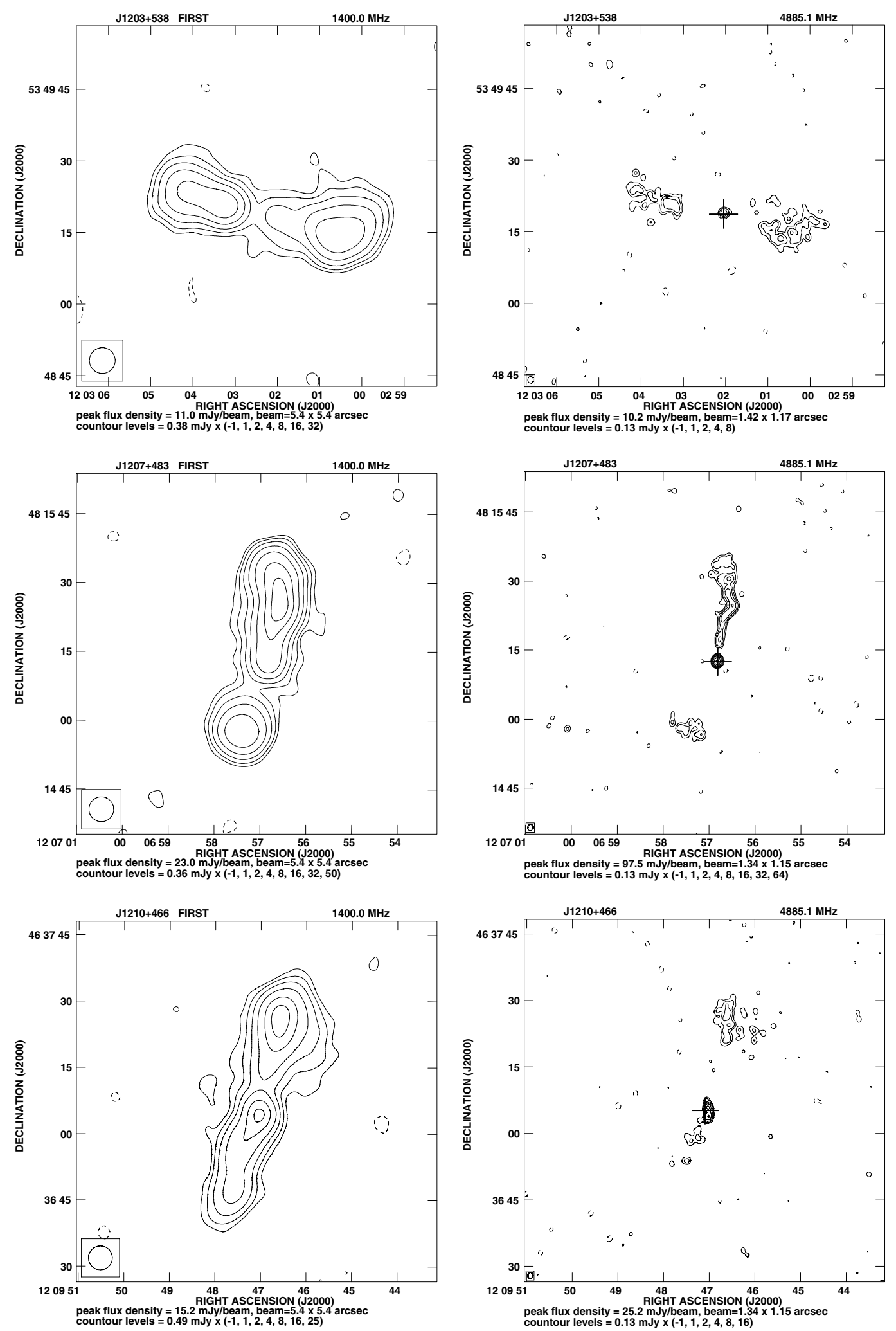

Fig. A.1. continued. 
M. P. Gawroński et al.: Hybrid morphology radio sources from the FIRST survey, Online Material p 7
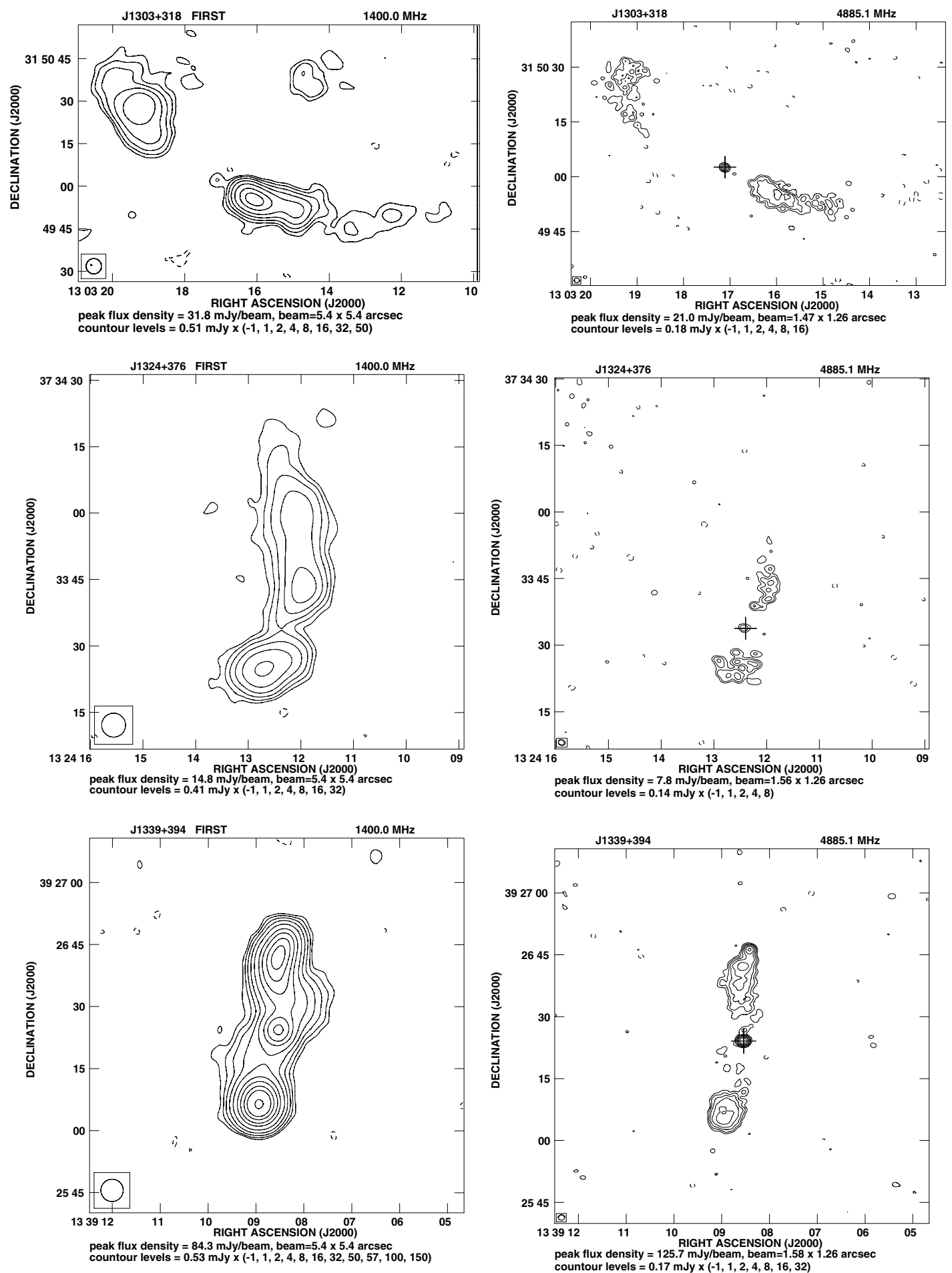

Fig. A.1. continued. 
M. P. Gawroński et al.: Hybrid morphology radio sources from the FIRST survey, Online Material p 8
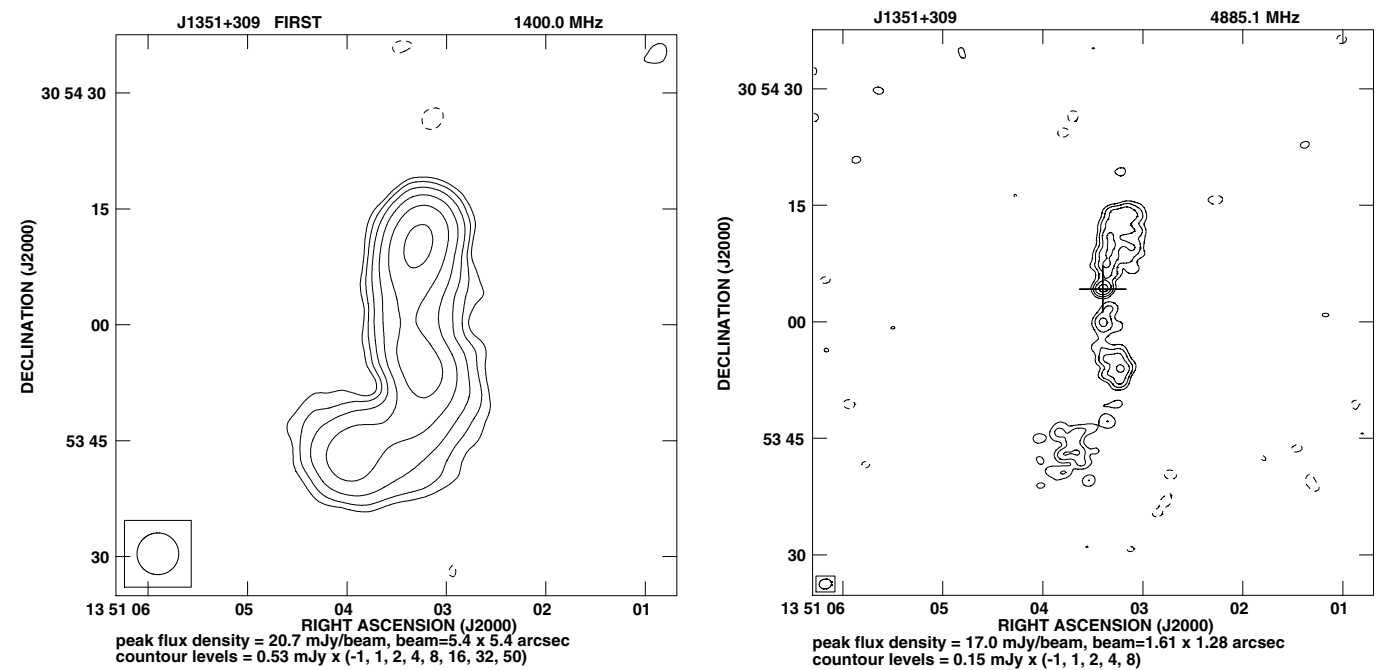

Fig. A.1. continued. 